\title{
Synthesis of Polysubstituted 4-Fluoroquinolinones
}

\author{
Alexander S. Kiselyov, Evgueni L. Piatnitski and \\ Jacqueline Doody
}

\section{Experimental Part}

In a typical experimental procedure: solution of 2-(trifluoromethyl)aniline (1) (161 $\mathrm{mg}, 1 \mathrm{mmol}$ ) in $5 \mathrm{~mL}$ of dry THF was added via syringe to a vigorously stirred mixture of arylacetate $(4 \mathrm{mmol})$ and LDA $(6 \mathrm{mmol}$, freshly prepared from diisopropylamine and $n$-BuLi) in $20 \mathrm{~mL}$ of dry $\mathrm{THF}$ at $-78^{\circ} \mathrm{C}$ under Ar. The mixture was allowed to warm to room temperature and stirred for an additional $4 \mathrm{~h}$. The resulting solution was concentrated in vacuo. The residue was partitioned between EtOAc and concentrated aqueous $\mathrm{NH}_{4} \mathrm{Cl}$, and the aqueous layer was extracted with EtOAc. Combined organic extracts were dried over $\mathrm{Na}_{2} \mathrm{SO}_{4}$, concentrated in vacuo, and purified by column chromatography (Silica gel, hexane/EtOAc $=1: 3$ ) to afford analytically pure quinolinones 3 .

4-Fluoro-3phenylhydroquinolin-2-one, 3a, $66 \%$ yield, m.p. $280^{\circ} \mathrm{C}$ (decomp.). ${ }^{1} \mathrm{H}$ NMR $\left(400 \mathrm{MHz}, \mathrm{dmso}_{6}\right): \delta 7.23(\mathrm{t}, J=7.6 \mathrm{~Hz}, 1 \mathrm{H}), 7.30-7.56(\mathrm{~m}, 6 \mathrm{H}), 7.64\left(\mathrm{dd}, J_{1}=7.6\right.$ $\left.\mathrm{Hz}, J_{2}=1.2 \mathrm{~Hz}, 1 \mathrm{H}\right), 7.82(\mathrm{~d}, J=7.6 \mathrm{~Hz}, 1 \mathrm{H}), 12.06$ (br s, exch. $\left.\mathrm{D}_{2} \mathrm{O}, 1 \mathrm{H}\right) ;{ }^{19} \mathrm{~F} \mathrm{NMR}$ (400 MHz, dmso-d 6 ): $\delta$-112.2; ESI MS: (M+1) 240, (M-1) 238. Elemental analysis, calcd. for $\mathrm{C}_{15} \mathrm{H}_{10}$ FNO: C, 75.30; H, 4.21; N, 5.85. Found: C, 75.13; H, 4.02; N, 5.61.

4-Fluoro-3-(3-pyridyl)hydroquinolin-2-one, 3b, 59\% yield, m.p. $280{ }^{\circ} \mathrm{C}$ (decomp.). ${ }^{1} \mathrm{H}$ NMR (400 MHz, dmso-d 6 ): $\delta 7.26(\mathrm{t}, J=7.6 \mathrm{~Hz}, 1 \mathrm{H}), 7.34$ (d,J=7.6 Hz, 1H), 7.43 (dd, $\left.J_{1}=7.6 \mathrm{~Hz}, J_{2}=1.2 \mathrm{~Hz}, 1 \mathrm{H}\right), 7.54(\mathrm{t}, J=7.6 \mathrm{~Hz}, 1 \mathrm{H}), 7.82(\mathrm{~d}, J=7.6 \mathrm{~Hz}, 1 \mathrm{H}), 8.02(\mathrm{~d}, J$ $=7.6 \mathrm{~Hz}, 1 \mathrm{H}), 8.64(\mathrm{~d}, J=1.2 \mathrm{~Hz}), 8.94(\mathrm{~s}, 1 \mathrm{H}), 11.94$ (br s, exch. $\left.\mathrm{D}_{2} \mathrm{O}, 1 \mathrm{H}\right) ;{ }^{19} \mathrm{~F} \mathrm{NMR}$ (400 MHz, dmso-d $\left.)_{6}\right): \delta$-111; ESI MS: (M+1) 241, (M-1) 239. Elemental analysis, calcd. for $\mathrm{C}_{14} \mathrm{H}_{9} \mathrm{FN}_{2} \mathrm{O}: \mathrm{C}, 69.99 ; \mathrm{H}, 3.78 ; \mathrm{N}, 11.66$. Found: C, 69.81; H, 3.86; N, 11.52. 
4-Fluoro-3-(4-methoxyphenyl)hydroquinolin-2-one, 3c, $71 \%$ yield, m.p. $280^{\circ} \mathrm{C}$ (decomp.). ${ }^{1} \mathrm{H}$ NMR (400 MHz, dmso-d 6 ): $\delta 3.86$ (s, 3H, OMe), 7.26 (t, $J=7.6 \mathrm{~Hz}, 1 \mathrm{H}$ ), $6.96(\mathrm{~d}, J=7.6 \mathrm{~Hz}, 2 \mathrm{H}), 7.14(\mathrm{~d}, J=7.6 \mathrm{~Hz}, 1 \mathrm{H}), 7.22\left(\mathrm{dd}, J_{1}=7.6 \mathrm{~Hz}, J_{2}=1.2 \mathrm{~Hz}, 1 \mathrm{H}\right)$, $7.58(\mathrm{~d}, J=7.6 \mathrm{~Hz}, 2 \mathrm{H}), 7.76(\mathrm{t}, J=7.6 \mathrm{~Hz}, 1 \mathrm{H}), 7.82(\mathrm{~d}, J=7.6 \mathrm{~Hz}, 1 \mathrm{H}), 12.04$ (br s, exch. $\left.\mathrm{D}_{2} \mathrm{O}, 1 \mathrm{H}\right) ;{ }^{13} \mathrm{C}$ NMR (100 MHz, dmso-d $\left.\mathrm{d}_{6}\right): \delta 114.2,115.6,118.8,122.6,123.4$, $123.8,126.8,133.1,139.2,149.2,151.3,160.2,161.7,163.7 ;{ }^{19} \mathrm{~F}$ NMR (400 MHz, dmso$\left.\mathrm{d}_{6}\right): \delta-110.8 ; \mathrm{ESI}$

MS: (M+1) 270, (M-1) 268. Elemental analysis, calcd. for $\mathrm{C}_{16} \mathrm{H}_{12} \mathrm{FNO}_{2}$ : C, 71.37; $\mathrm{H}$, 4.49; N, 5.20. Found: C, 71.15; H, 4.54; N, 5.02.

3-(3,5-dimethoxyphenyl)-4-fluorohydroquinolin-2-one, 3d, $63 \%$ yield, m.p. $280^{\circ} \mathrm{C}$ (decomp.). ${ }^{1} \mathrm{H}$ NMR (400 MHz, dmso-d ${ }_{6}, 25^{\circ} \mathrm{C}$ ): $\delta 3.64$ (s, 3H, OMe), 3.68 (s, 3H, OMe), 6.82-7.06 (m, 3H), $7.12(\mathrm{t}, J=7.6 \mathrm{~Hz}, 1 \mathrm{H}), 7.26\left(\mathrm{dd}, J_{1}=7.6 \mathrm{~Hz}, J_{2}=1.2 \mathrm{~Hz}, 1 \mathrm{H}\right), 7.42$ (t, $J=7.6 \mathrm{~Hz}, 1 \mathrm{H}), 7.64(\mathrm{~d}, J=7.6 \mathrm{~Hz}, 1 \mathrm{H}), 11.86$ (br s, exch. $\left.\mathrm{D}_{2} \mathrm{O}, 1 \mathrm{H}\right) ;{ }^{13} \mathrm{C}$ NMR $(100$ MHz, dmso-d ${ }_{6}$ ): $\delta 114.2,115.6,118.8,122.6,123.4,123.8,126.8,133.1,139.2,149.2$, 151.3, 160.2, 161.7, 163.7; ${ }^{19} \mathrm{~F}$ NMR (400 MHz, dmso-d $\left.\mathrm{d}_{6}\right): \delta$-110.6; ESI MS: $(\mathrm{M}+1)$ 300, (M-1) 298. Elemental analysis, calcd. for $\mathrm{C}_{17} \mathrm{H}_{14} \mathrm{FNO}_{3}$ : C, 68.22; H, 4.71; N, 4.68. Found: C, 67.96; H, 4.84; N, 4.39.

4-Fluoro-3-(2-thienyl)hydroquinolin-2-one, 3e, 58\% yield, m.p. $280^{\circ} \mathrm{C}$ (decomp.). ${ }^{1} \mathrm{H}$ NMR (400 MHz, dmso-d 6 ): $\delta 6.98$ (dd, $\left.J_{1}=7.6 \mathrm{~Hz}, J_{2}=1.2 \mathrm{~Hz}, 1 \mathrm{H}\right), 7.04(\mathrm{t}, J=7.6 \mathrm{~Hz}$, $1 \mathrm{H}), 7.18\left(\mathrm{~d}, J_{1}=7.6 \mathrm{~Hz}, 1 \mathrm{H}\right), 7.40(\mathrm{t}, J=7.6 \mathrm{~Hz}, 1 \mathrm{H}), 7.52(\mathrm{~d}, J=1.2 \mathrm{~Hz}, 1 \mathrm{H}), 7.64(\mathrm{~d}$, $J=7.6 \mathrm{~Hz}, 1 \mathrm{H}), 7.78(\mathrm{~d}, J=7.6 \mathrm{~Hz}, 1 \mathrm{H}), 11.96$ (br s, exch. $\left.\mathrm{D}_{2} \mathrm{O}, 1 \mathrm{H}\right) ;{ }^{19} \mathrm{~F}$ NMR $(400$ MHz, dmso-d 6 ): $\delta$-110.8; ESI MS: (M+1) 246, (M-1) 244. Elemental analysis, calcd. for $\mathrm{C}_{13} \mathrm{H}_{8}$ FNOS: C, 63.66; H, 3.29; N, 5.71. Found: C, 63.30; H, 3.38; N, 5.58.

3-(3,4-dichlorophenyl)-4-fluorohydroquinolin-2-one, 3f, $72 \%$ yield, m.p. $280^{\circ} \mathrm{C}$ (decomp.). ${ }^{1} \mathrm{H}$ NMR $\left(400 \mathrm{MHz}\right.$, dmso-d $\left._{6}, 25^{\circ} \mathrm{C}\right): \delta 7.15(\mathrm{t}, J=7.6 \mathrm{~Hz}, 1 \mathrm{H}), 7.22\left(\mathrm{dd}, J_{1}=\right.$ $\left.7.6 \mathrm{~Hz}, J_{2}=1.2 \mathrm{~Hz}, 1 \mathrm{H}\right), 7.33(\mathrm{~d}, J=7.6 \mathrm{~Hz}, 1 \mathrm{H}), 7.42(\mathrm{t}, J=7.6 \mathrm{~Hz}, 1 \mathrm{H}), 7.68(\mathrm{~d}, J=$ $7.6 \mathrm{~Hz}, 1 \mathrm{H}), 7.75(\mathrm{~m}, 2 \mathrm{H}), 12.08$ (br s, exch. $\left.\mathrm{D}_{2} \mathrm{O}, 1 \mathrm{H}\right) ;{ }^{19} \mathrm{~F}$ NMR (400 MHz, dmso-d 6 ): $\delta$ 
-112.2; ESI MS: (M+1) 309, (M-1) 307. Elemental analysis, calcd. for $\mathrm{C}_{15} \mathrm{H}_{8} \mathrm{Cl}_{2} \mathrm{FNO}$ : C, $58.47 ; \mathrm{H}, 2.62 ; \mathrm{N}, 4.55$. Found: C, 58.36; H, 2.50; N, 4.36.

3-[(3,4-demethoxyphenyl)methyl]-4-fluorohydroquinolin-2-one, $\mathbf{3 g}, 52 \%$ yield, m.p. $280^{\circ} \mathrm{C}$ (decomp.). ${ }^{1} \mathrm{H}$ NMR (400 MHz, dmso-d ${ }_{6}$ ): $\delta 3.82$ (s, 6H, 2OMe), 3.92 (s, $2 \mathrm{H}$, $\left.\mathrm{CH}_{2}\right), 6.73(\mathrm{t}, J=7.6 \mathrm{~Hz}, 1 \mathrm{H}), 6.91\left(\mathrm{dd}, J_{1}=7.6 \mathrm{~Hz}, J_{2}=1.2 \mathrm{~Hz}, 1 \mathrm{H}\right), 6.96(\mathrm{~s}, 1 \mathrm{H}), 7.48(\mathrm{t}$, , $J=7.6 \mathrm{~Hz}, 1 \mathrm{H}), 7.62(\mathrm{~d}, J=7.6 \mathrm{~Hz}, 1 \mathrm{H}), 7.74(\mathrm{t},, J=7.6 \mathrm{~Hz}, 1 \mathrm{H}), 7.83(\mathrm{~d}, J=7.6 \mathrm{~Hz}$, 1H), 11.98 (br s, exch. $\mathrm{D}_{2} \mathrm{O}, 1 \mathrm{H}$ ); ${ }^{19} \mathrm{~F}$ NMR (400 MHz, dmso-d 6 ): $\delta$-110.2; ESI MS: (M+1) 314, (M-1) 312. Elemental analysis, calcd. for $\mathrm{C}_{18} \mathrm{H}_{16} \mathrm{FNO}_{3}$ : C, 69.00; H, 5.15; N, 4.47. Found: C, 68.81; H, 5.24; N, 4.28.

4-Fluoro-3-(3-pyridyl)hydroquinolin-2-one, 3h, 59\% yield, m.p. $280^{\circ} \mathrm{C}$ (decomp.). ${ }^{1} \mathrm{H}$ NMR (400 MHz, dmso-d $\left.{ }_{6}\right): \delta 7.26-7.42(\mathrm{~m}, 4 \mathrm{H}), 7.45(\mathrm{~d}, J=7.6 \mathrm{~Hz}, 1 \mathrm{H}), 7.69\left(\mathrm{dd}, J_{1}=\right.$ $\left.7.6 \mathrm{~Hz}, J_{2}=1.2 \mathrm{~Hz}, 1 \mathrm{H}\right), 7.83(\mathrm{~d}, J=7.6 \mathrm{~Hz}, 1 \mathrm{H}), 12.13$ (br s, exch. $\left.\mathrm{D}_{2} \mathrm{O}, 1 \mathrm{H}\right) ;{ }^{19} \mathrm{~F} \mathrm{NMR}$ (400 MHz, dmso-d $\left.\mathrm{d}_{6}\right): \delta$-112.8; ESI MS: $(\mathrm{M}+1)$ 276, (M-1) 274. Elemental analysis, calcd. for $\mathrm{C}_{15} \mathrm{H}_{8} \mathrm{~F}_{3} \mathrm{NO}$ : C, 65.46; H, 2.93; N, 5.09. Found: C, 65.34; H, 2.82; N, 4.93.

4,6-Difluoro-3-(3-pyridyl)hydroquinolin-2-one, 3i, $72 \%$ yield, m.p. $280^{\circ} \mathrm{C}$ (decomp.). ${ }^{1} \mathrm{H}$ NMR (400 MHz, dmso-d 6 ): $\delta$ 7.56-7.82 (m, 4H), $8.02(\mathrm{~d}, J=7.6 \mathrm{~Hz}, 1 \mathrm{H}), 8.70$ (d, $J$ $=1.2 \mathrm{~Hz}, 1 \mathrm{H}), 8.84(\mathrm{~s}, 1 \mathrm{H}), 12.52\left(\right.$ br s, exch. $\left.\mathrm{D}_{2} \mathrm{O}, 1 \mathrm{H}\right) ;{ }^{19} \mathrm{~F}$ NMR $\left(400 \mathrm{MHz}\right.$, dmso-d $\left.\mathrm{d}_{6}\right)$ : $\delta$-113.5; ESI MS: (M+1) 259, (M-1) 257. Elemental analysis, calcd. for $\mathrm{C}_{14} \mathrm{H}_{8} \mathrm{~F}_{2} \mathrm{~N}_{2} \mathrm{O}: \mathrm{C}$, 65.12; H, 3.12; N, 10.85. Found: C, 64.84; H, 3.24; N, 10.61.

4,6-Difluoro-3-(1-naphthyl)hydroquinolin-2-one, 3j, 77\% yield, m.p. $280^{\circ} \mathrm{C}$ (decomp.). ${ }^{1} \mathrm{H}$ NMR (400 MHz, dmso-d $\left.{ }_{6}\right): \delta$ 7.32-7.72 (m, 7H), $7.76(\mathrm{~s}, 1 \mathrm{H}), 7.92-7.94(\mathrm{~m}, 2 \mathrm{H})$, 12.14 (br s, exch. $\left.\mathrm{D}_{2} \mathrm{O}, 1 \mathrm{H}\right) ;{ }^{19} \mathrm{~F}$ NMR (400 MHz, dmso-d 6 ): $\delta$-111.7; ESI MS: $(\mathrm{M}+1)$ 308, (M-1) 307. Elemental analysis, calcd. for $\mathrm{C}_{19} \mathrm{H}_{11} \mathrm{~F}_{2} \mathrm{NO}$ : C, 74.26; H, 3.61; N, 4.56. Found: C, 74.02; H, 3.45; N, 4.31.

4,6-Difluoro-3-(3-pyridyl)hydroquinolin-2-one, 3k, 78\% yield, m.p. $280^{\circ} \mathrm{C}$ (decomp.). ${ }^{1} \mathrm{H}_{\mathrm{NMR}}\left(400 \mathrm{MHz}\right.$, dmso-d $\left._{6}\right): \delta$ 7.30-7.65 (m, 5H), $7.82(\mathrm{~d}, J=7.6 \mathrm{~Hz}, 1 \mathrm{H}), 8.64(\mathrm{~d}, J$ 
$=2.4 \mathrm{~Hz}, 1 \mathrm{H}), 12.26$ (br s, exch. $\left.\mathrm{D}_{2} \mathrm{O}, 1 \mathrm{H}\right) ;{ }^{19} \mathrm{~F}$ NMR $\left(400 \mathrm{MHz}, \mathrm{dmso}^{-\mathrm{d}_{6}}\right): \delta-113.2$; ESI MS: (M+1) 259, (M-1) 257. Elemental analysis, calcd. for $\mathrm{C}_{14} \mathrm{H}_{8} \mathrm{~F}_{2} \mathrm{~N}_{2} \mathrm{O}$ : C, 65.12; $\mathrm{H}$, 3.12; N, 10.85. Found: C, 64.88; H, 3.27; N, 10.62 .

3-(3,5-Dimethoxyphenyl)-4,6-difluorohydroquinolin-2-one, 31, 74\% yield, m.p. $280^{\circ} \mathrm{C}$ (decomp.). ${ }^{1} \mathrm{H}$ NMR (400 MHz, dmso-d $\mathrm{d}_{6}, 25^{\circ} \mathrm{C}$ ): $\delta 3.98$ (s, 3H, OMe), 4.04 (s, 3H, OMe), 7.12-7.28 (m, 2H), $7.30(\mathrm{~s}, 1 \mathrm{H}), 7.54-7.80(\mathrm{~m}, 3 \mathrm{H}), 12.28$ (br s, exch. $\left.\mathrm{D}_{2} \mathrm{O}, 1 \mathrm{H}\right) ;{ }^{19} \mathrm{~F}$ NMR (400 MHz, dmso-d ${ }_{6}$ ): $\delta$-112.5; ESI MS: (M+1) 318, (M-1) 316. Elemental analysis, calcd. for $\mathrm{C}_{17} \mathrm{H}_{13} \mathrm{~F}_{2} \mathrm{NO}_{3}$ : C, 64.35; H, 4.13; N, 4.41. Found: C, 64.06; H, 4.22; $\mathrm{N}, 4.23$.

4,6-Difluoro-3-(2-thienyl)hydroquinolin-2-one, 3m, 52\% yield, m.p. $280^{\circ} \mathrm{C}$ (decomp.). ${ }^{1} \mathrm{H}$ NMR (400 MHz, dmso-d $\left.{ }_{6}\right): \delta 7.48(\mathrm{t}, J=7.6 \mathrm{~Hz}, 1 \mathrm{H}), 7.58-7.64(\mathrm{~m}, 2 \mathrm{H}), 7.79(\mathrm{dd}, J$ $\left.{ }_{1}=7.6 \mathrm{~Hz}, J_{2}=1.2 \mathrm{~Hz}, 1 \mathrm{H}\right), 7.88\left(\mathrm{dd}, J_{1}=7.6 \mathrm{~Hz}, J_{2}=1.2 \mathrm{~Hz}, 1 \mathrm{H}\right), 8.02(\mathrm{~d}, J=1.2 \mathrm{~Hz}$, $1 \mathrm{H}), 12.34$ (br s, exch. $\mathrm{D}_{2} \mathrm{O}, 1 \mathrm{H}$ ); ${ }^{19} \mathrm{~F}$ NMR (400 MHz, dmso-d 6 ): $\delta$-112.6; ESI MS: $(\mathrm{M}+1)$ 264, (M-1) 262. Elemental analysis, calcd. for $\mathrm{C}_{13} \mathrm{H}_{7} \mathrm{~F}_{2} \mathrm{NOS}$ : C, 59.31; H, 2.68; N, 5.32. Found: C, 59.06; H, 2.49; N, 5.13.

4,6-Difluoro-3-(4-methoxyphenyl)hydroquinolin-2-one, 3n, 57\% yield, m.p. $280^{\circ} \mathrm{C}$ (decomp.). ${ }^{1} \mathrm{H}$ NMR (400 MHz, dmso-d 6 ): $\delta 3.78$ (s, 3H, OMe), 6.90 (d, $J=7.6 \mathrm{~Hz}, 2 \mathrm{H}$ ), 7.30-7.66 (m, 3H), 7.54 (d, $J=7.6 \mathrm{~Hz}, 2 \mathrm{H}), 12.03$ (br s, exch. $\left.\mathrm{D}_{2} \mathrm{O}, 1 \mathrm{H}\right) ;{ }^{19} \mathrm{~F}$ NMR (400 MHz, dmso-d 6 ): $\delta$-111.4; ESI MS: (M+1) 288, (M-1) 286. Elemental analysis, calcd. for $\mathrm{C}_{16} \mathrm{H}_{11} \mathrm{~F}_{2} \mathrm{NO}_{2}$ : C, 66.90; H, 3.86; N, 4.88. Found: C, 66.76; H, 3.95; N, 4.69.

3-(3,4-Dichlorophenyl)-4,8-difluorohydroquinolin-2-one, 3o, $79 \%$ yield, m.p. $280^{\circ} \mathrm{C}$ (decomp.). ${ }^{1} \mathrm{H}$ NMR (400 MHz, dmso-d 6 ): $\delta 7.29$ (m, 1H), 7.33-7.40 (m, 2H), 7.64 (dd, $J$ $\left.{ }_{1}=7.6 \mathrm{~Hz}, J_{2}=1.2 \mathrm{~Hz}, 1 \mathrm{H}\right), 7.76(\mathrm{~d}, J=$

$7.6 \mathrm{~Hz}, 1 \mathrm{H}), 7.85(\mathrm{~s}, 1 \mathrm{H}), 12.28$ (br s, exch. $\left.\mathrm{D}_{2} \mathrm{O}, 1 \mathrm{H}\right) ;{ }^{19} \mathrm{~F}$ NMR (400 MHz, dmso-d 6 ): $\delta$ -112.6; ESI MS: (M+1) 327, (M-1) 325. Elemental analysis, calcd. for $\mathrm{C}_{15} \mathrm{H}_{7} \mathrm{Cl}_{2} \mathrm{~F}_{2} \mathrm{NO}$ : C, 55.24; H, 2.16; N, 4.29. Found: C, 55.02; H, 2.27; N, 4.11. 
4,8-Difluoro-3-(1-naphthyl)hydroquinolin-2-one, $3 p, \quad 77 \%$ yield, m.p. $\quad 280^{\circ} \mathrm{C}$ (decomp.). ${ }^{1} \mathrm{H}$ NMR (400 MHz, dmso-d 6 ): $\delta 7.22$ (m, 1H), 7.40-7.66 (m, 6H), 7.69 (dd, J $\left.{ }_{1}=7.6 \mathrm{~Hz}, J_{2}=1.2 \mathrm{~Hz}, 1 \mathrm{H}\right), 8.01(\mathrm{~m}, 2 \mathrm{H}), 12.20$ (br s, exch. $\left.\mathrm{D}_{2} \mathrm{O}, 1 \mathrm{H}\right) ;{ }^{19} \mathrm{~F}$ NMR $(400$ MHz, dmso-d $\left.{ }_{6}\right): \delta$-112.0; ESI MS: (M+1) 308, (M-1) 306. Elemental analysis, calcd. for $\mathrm{C}_{19} \mathrm{H}_{11} \mathrm{~F}_{2} \mathrm{NO}$ : C, 74.26; H, 3.61; N, 4.56. Found: C, 74.03; H, 3.48; N, 4.33.

6-Bromo-4-fluoro-3-(4-methoxyphenyl)hydroquinolin-2-one, 3q, 63\% yield, m.p. $280^{\circ} \mathrm{C}$ (decomp.). ${ }^{1} \mathrm{H}$ NMR (400 MHz, dmso-d 6 ): $\delta 3.75$ (s, 3H, OMe), 6.96 (d, $J=7.6$ $\mathrm{Hz}, 2 \mathrm{H}), 7.22(\mathrm{~d}, J=7.6 \mathrm{~Hz}, 1 \mathrm{H}), 7.42(\mathrm{~d}, J=7.6 \mathrm{~Hz}, 2 \mathrm{H}), 7.56(\mathrm{~d}, J=7.6 \mathrm{~Hz}, 1 \mathrm{H}), 7.82$ (s, 1H), 12.01 (br s, exch. $\left.\mathrm{D}_{2} \mathrm{O}, 1 \mathrm{H}\right) ;{ }^{19} \mathrm{~F}$ NMR (400 MHz, dmso-d 6 ): $\delta$-111.2; ESI MS: (M+1) 349, (M-1) 347. Elemental analysis, calcd. for $\mathrm{C}_{16} \mathrm{H}_{11} \mathrm{BrFNO}_{2}$ : C, 55.20; H, 3.18; N, 4.02. Found: C, 54.94; H, 2.96; N, 3.73.

6-Bromo-4-fluoro-3-(3-thienyl)hydroquinolin-2-one, 3r, $55 \%$ yield, m.p. $280^{\circ} \mathrm{C}$ (decomp.). ${ }^{1} \mathrm{H}$ NMR (400 MHz, dmso-d 6 ): $\delta 7.01$ (dd, $\left.J_{1}=7.6 \mathrm{~Hz}, J_{2}=1.2 \mathrm{~Hz}, 1 \mathrm{H}\right), 7.13$ $(\mathrm{d}, J=7.6 \mathrm{~Hz}, 1 \mathrm{H}), 7.52-7.66(\mathrm{~m}, 2 \mathrm{H}), 7.76(\mathrm{~s}$,

1H), 7.82 (s, 1H), 12.16 (br s, exch. $\left.\mathrm{D}_{2} \mathrm{O}, 1 \mathrm{H}\right) ;{ }^{19} \mathrm{~F}$ NMR (400 MHz, dmso-d 6 ): $\delta$-111.9; ESI MS: (M+1) 325, (M-1) 323. Elemental analysis, calcd. for $\mathrm{C}_{13} \mathrm{H}_{7} \mathrm{BrFNOS}$ : C, 48.17; H, 2.18; N, 4.32. Found: C, 48.04; H, 2.06; N, 4.20.

6-Bromo-4-fluoro-3-(2-thienyl)hydroquinolin-2-one, 3s, $61 \%$ yield, m.p. $280^{\circ} \mathrm{C}$ (decomp.). ${ }^{1} \mathrm{H}$ NMR (400 MHz, dmso-d 6 ): $\delta 7.21$ (d, $\left.J=7.6 \mathrm{~Hz}, 1 \mathrm{H}\right), 7.43$ (d, $J=1.2 \mathrm{~Hz}$, $1 \mathrm{H}), 7.48(\mathrm{~d}, J=1.2 \mathrm{~Hz}, 1 \mathrm{H}), 7.67\left(\mathrm{dd}, J_{1}=7.6 \mathrm{~Hz}, J_{2}=1.2 \mathrm{~Hz}, 1 \mathrm{H}\right), 7.86(\mathrm{~s}, 1 \mathrm{H}), 8.06$ (s, 1H), 12.20 (br s, exch. $\left.\mathrm{D}_{2} \mathrm{O}, 1 \mathrm{H}\right) ;{ }^{19} \mathrm{~F}$ NMR (400 MHz, dmso-d 6 ): $\delta$-112.2; ESI MS: $(\mathrm{M}+1)$ 325, (M-1) 323. Elemental analysis, calcd. for $\mathrm{C}_{13} \mathrm{H}_{7}$ BrFNOS: C, 48.17; H, 2.18; N, 4.32. Found: C, 47.89; H, 2.02; N, 4.11.

6-Bromo-4-fluoro-3-(4-pyridyl)hydroquinolin-2-one, 3t, $81 \%$ yield, m.p. $280^{\circ} \mathrm{C}$ (decomp.). ${ }^{1} \mathrm{H}$ NMR (400 MHz, dmso-d 6 ): 7.22 (d, $\left.J=7.6 \mathrm{~Hz}, 1 \mathrm{H}\right), 7.48$ (d, J=7.6 Hz, 
2H), 7.64 (d, $J=7.6 \mathrm{~Hz}, 1 \mathrm{H}), 7.83$ (s, 1H), 8.61 (d, $J=7.6 \mathrm{~Hz}, 2 \mathrm{H}), 12.18$ (br s, exch.

$\left.\mathrm{D}_{2} \mathrm{O}, 1 \mathrm{H}\right) ;{ }^{19} \mathrm{~F}$ NMR $\left(400 \mathrm{MHz}, \mathrm{dms}_{6}-\mathrm{d}_{6}\right): \delta$-112.8; ESI MS: (M+1) 320, (M-1) 318.

Elemental analysis, calcd. for $\mathrm{C}_{14} \mathrm{H}_{8} \mathrm{BrFN}_{2} \mathrm{O}$ : C, 52.69; H, 2.53; N, 8.78. Found: C, 52.52; H, 2.63; N, 8.57. 